\title{
Second-generation antipsychotics - a panacea?
}

\author{
Paul D Carey is a research psychiatrist in the MRC Research Unit on \\ Anxiety Disorders, Department of Psychiatry, Stellenbosch University. \\ His research interests include psychopharmacology and brain imag- \\ ing with application to psychiatry. Dan Stein is Director of the Unit. His \\ main research interests include the psychobiology and psychophar- \\ macology of anxiety disorders. Robin A Emsley is Professor of \\ Psychiatry at Stellenbosch University; his main research interests \\ include the psychopharmacology of schizophrenia.
}

The introduction of the second-generation antipsychotics (SGAs) (atypical antipsychotics) for the treatment of psychotic disorders has changed the goals of modern therapeutic outcomes. No longer are positive symptoms of psychosis in schizophrenia the only outcomes measured, but dimensions including negative and cognitive symptoms are now crucial to determining efficacy. As a result outcomes in these areas are increasingly impacting on our choice of treatment.

The initial and most visible benefits of the SGAs were good tolerability, particularly reduced risk of extra-pyramidal symptoms. This has resulted in widespread use of these compounds for a range of non-psychotic conditions. Prescribing habits for SGAs of general practitioners and psychiatrists in South Africa and the USA suggest that in excess of $70 \%$ of SGAs prescribed are for off-label indications. This is supported in part by a now considerable body of open-label evidence. Medical practitioners have, with trial and error, always pushed conventional boundaries and pursued alternative treatments for complex cases while weighing up potential risks and benefits. However, present prescribing habits for SGAs possibly pose the risk of these drugs being branded a panacea for all 'difficult to treat' conditions. The practice is compounded by the relative lack of available pharmacotherapeutic options for those failing to respond to first-line treatments. Evidence-based medicine requires that we raise the questions of (i) whether there is any good evidence for efficacy of SGAs for non-psychotic indications; and (ii) the evidence regarding risk.

Mood and anxiety disorders are consistently found to be the most prevalent psychiatric disorders ${ }^{2}$ and are among the most burdensome of all medical conditions. ${ }^{3}$ Treatment is often difficult as reflected in remission rates in clinical trials generally being less than 50\% with 'resistance' in some 10 $50 \%$ of patients. Thus, treatment-refractory mood and anxiety disorders are a significant burden in the clinic, and it is probably not surprising that clinicians have used SGAs as alternative and accessible forms of treatment in this group.

Currently more than 15 studies provide positive open-label data on use of SGAs to augment conventional antidepressant therapy for treatment-refractory non-psychotic MDD. To date, however, there is still only a single published controlled study in this area demonstrating efficacy for the use of the combination of olanzapine and fluoxetine. ${ }^{4}$ In anxiety disorders, augmentation of treatment for obsessivecompulsive disorder has enjoyed most research attention with some 14 open-label augmentation studies (1 negative) and 6 controlled studies (2 negative) now published. For post traumatic stress disorder, more than five open-label studies and two controlled studies have shown positive outcomes. Even more limited data are available for generalised anxiety, social anxiety and panic disorders.

The relative lack of controlled data in these areas comes as somewhat of a surprise. Reasons for this may include (i) a lack of will on the part of non-industry funders to support this work; (ii) a lack of will on the part of clinicians/researchers to answer this question by building on the preliminary data; (iii) clinician experience that this is indeed a helpful treatment strategy and in their mind requires no further controlled evidence; and (iv) in the absence of clear indications from open-label data that a particular compound in this class is likely to be superior to its 'classmates', the commercial incentive for support is lost to the pharmaceutical industry. 
The last reason is likely to account for the general lack of research on augmentation strategies in refractory psychiatric conditions. This is likely to be compounded by the knowledge that the compounds are already being widely used for many off-label indications.

For the range of disorders for which SGAs have been tested in psychiatry, 5,6 less than 10\% of studies are double-blind and randomised. ${ }^{7}$ In excess of $90 \%$ of published open-label studies report positive findings. We should guard against the obvious pitfalls of drawing premature conclusions on efficacy from these data and remain aware of factors such as publication and file-drawer bias in favour of positive findings. With respect to controlled data on use of SGAs for a variety of indications, a negative finding has been reported for alcohol dependence (1 study), positive effects on certain behavioural aspects of pervasive developmental disorders 13 studies), positive results in Tourette's disorder (2 studies), and positive findings for behavioural and emotional symptoms central to borderline personality disorder (1 study). Further caution is appropriate when interpreting single studies with generally small sample sizes. Few would surely disagree that the many factors given above are likely to continue limiting opportunities to answer these questions in the future. This is unfortunate as progress on investigating the possibly distinct neurobiology of treatment-refractory disorders across the clinical spectrum will be slowed considerably.

The growing use of SGAs in the face of mounting data on their potential metabolic consequences is surprising. Sideeffects are well known and important, and should be particularly carefully considered when making treatment choices for off-label indications.

Metabolic consequences that should be the focus of attention include the effects on weight (gain) and the relationship, direct or indirect, with increased rates of insulin resistance, new-onset type 2 diabetes and dyslipidaemias. It is increasingly clear that the pharmacological profile of SGAs is more dissimilar than it is similar. Not suprising then is the similarly variable effect on weight. Two questions emerge. First, do patients with conditions (primarily psychotic) treated with these agents have a greater risk of developing obesity and type 2 diabetes without the effect of medication, and second, to what extent do these agents increase the rates of obesity and type 2 diabetes?

At present very few data are available on the pre-treatment characteristics of patients to help us answer the first question. Although untested, it seems likely that schizophrenia and other chronic mental illnesses have a negative (riskenhancingl effect on lifestyle that accompanies downward social drift, conferring a state-dependent risk. This is probably mediated by lower levels of physical activity, less nutritious diets, and higher rates of cigarette smoking and substance abuse. Certainly the converse, that psychiatric conditions offer some protection against these factors, seems highly improbable.

The question of weight gain with treatment appears to be the less vexing of the two. Significant weight gain can be induced by certain SGAs; however, there is considerable variability within the class. Conversely, to answer the question of risk of inducing type 2 diabetes and dyslipidaemias is more difficult given the lack of long-term prospective data in most treatment studies on psychiatric populations. It is well established that in normal populations moderate $(5 \mathrm{~kg})$ to severe $(15 \mathrm{~kg})$ increases in weight are accompanied by 2 -and 6 -fold increases in risk for type 2 diabetes respectively. As such the early and often remarkable weight gain seen in some patients would seem to confer significantly higher risk levels. Furthermore, there are some indications that increases in cholesterol may occur early in the course of treatment independent of weight gain. This suggests a direct drug effect, though for the majority of cases it is a physiological consequence of the weight gain.

The worldwide preoccupation with weight loss and the endless number of solutions to the pandemic that obesity is, suggest that lasting solutions remain elusive. In general, prevention of weight gain would seem to be the more appropriate response. This will require the evaluation of all prosepctive users of SGAs for risk factors including family history of obesity, diabetes and dyslipidaemia. It seems likely that guidelines will eventually emerge that will inform our choice of treatment based in part on the metabolic risk profile of individual patients. As a more general measure, metabolic monitoring guidelines for the use of SGAs have recently been published with recommendations on monitoring weight, BMI, blood pressure, blood glucose and lipid profile. The increases in risk with even moderate (> $5 \mathrm{~kg}$ ) weight gain should prompt a clear re-appraisal of the risk/benefit ratio for a compound in a particular individual. In this instance it may be useful to switch to an apparently more weight-neutral alternative, although data in this area are sparse. 
In conclusion, the high rate of failure of first-line treatments for mood and anxiety disorders clearly calls for further research. Certainly, preliminary evidence for the use of SGAs is promising and deserves future attention in refractory mood and anxiety disorders. The present lack of controlled data for use of SGAs for off-label indications, combined with a clear need to monitor potential metabolic changes induced by these compounds, suggests that this work should probably be conducted in specialist centres. Despite this lack of evidence, off-label use of this class will continue, but should be with appropriate caution. A clear appraisal of the individual patient's metabolic risk, weighed against the evidence for a clinical response in a paticular condition, should be made. Equally crucial is the need to inform patients of the potential for metabolic consequences and seek their informed consent for the intervention. These steps will enhance the quality of the therapeutic alliance and in no way diminish the physician's role in crafting a treatment strategy for those

Alonso f Angermeyer MC, Bernert S, et al. Prevalence of mental disorders in Europe: results from the European Study of he Epidemiology of Mental Disorders (ESEMeD) project. Acta Psychiatr Scand Suppl 2004; 420: $21-27$

2. Demyttenaere K, Bruffaerts R, Posada-Villa J et al. Prevalence, severity and unme need for treatment of mental disorders in he World Heal h Organization World Mental Health Surveys. JAMA 2004; 291: 258 1-2590

3. Alonso J, Angermeyer MC, Bernert $S$, et al. Disability and quality of life impact of mental disorders in Europe: results from he European Study of the Epidemiology of Mental Disorders (ESEMeD) project. Acta Psychiatr Scand Suppl 2004; 420: 38-46. patients refractory to conventional therapies. In fact this should remain the essence of clinical practice and may frequently justify the use of these and other novel treatment strategies.

\section{Paul D Carey}

\section{Dan Stein}

MRC Research Unit on Anxiety Disorders

Department of Psychiatry

Stellenbosch University

Stellenbosch, W Cape

\section{Robin Emsley}

Department of Psychiatry Stellenbosch University

Stellenbosch, W Cape

4. Shelton RC, Tollefson GD, Tohen $M$, et al. A novel augmentation strategy for treating resistant major depression. Am J Psychiatry 2001; 158: 131-134

5. Buckley PF. Broad therapeutic uses of atypical antipsychotic medications. Biol Psychiatry 2001; 50: $912-924$

6. Glick ID, Murray SR, Vasudevan P, Marder SR, Hu RJ. Treatment wi h atypical antipsychotics: new indications and new populations. J Psychiatr Res 200 1 35: 187 191

7. Fountoulakis KN, Nimatoudis I, lacovides A, Kaprinis G. Off-label indications for atypical antipsychotics: A systematic review. Ann Gen Hosp Psychiatry 2004; 3: 4 